\title{
Design of active structural acoustic control systems by eigenproperty assignment
}

\author{
Ricardo A. Burdisso and Chris R. Fuller \\ Vibration and Acoustic Laboratories, Mechanical Engineering Department, Virginia Polytechnic Institute \\ and State University, Blacksburg, Virginia 24061-0238
}

(Received 9 June 1993; revised 8 March 1994; accepted 22 April 1994)

\begin{abstract}
The traditional design approach of feedforward control systems involves the selection of number and location of the actuators and sensors based on some physical understanding of the system. This empirical methodology yields satisfactory results for simple structures and sinusoidal inputs. However, such a heuristic approach can easily result in an inefficient control system with an unnecessarily large number of control channels for complex structures and more realistic disturbances. In this work an efficient formulation is presented for the design of actuators and sensors for structurally radiated sound reduction. The technique is based on the modification of the eigenstructure such that the system responds with the weakest set of modal radiators. The technique is applicable to both narrow-band and broadband excitations. The formulation is demonstrated for controlling the odd-odd modes of a simply supported plate driven by a point force located at the center of the plate. The radiation due to the first three odd-odd modes is reduced with a single-input, single-output (SISO) controller. The control actuator and error sensor are implemented with piezoelectric (PZT) ceramics and polyvinylidene fluoride (PVDF) films, respectively. It is shown that the design approach yields excellent global sound reduction.
\end{abstract}

PACS numbers: $43.40 . \mathrm{Vn}$

\section{INTRODUCTION}

Sound radiated by vibrating structures is a persistent problem in numerous industrial applications, and it has long been a subject of research interest in the acoustic community. The common practice of using passive techniques often results in heavy systems that are inefficient at low frequencies. In recent years, considerable effort has been devoted to active control techniques to reduce low-frequency structurally radiated sound. The understanding of the physics of the problem has yielded efficient control strategies. One such approach has been proposed by Fuller ${ }^{1}$ in which the control inputs are applied directly to the vibrating structure while minimizing radiated sound or related variable. This technique, known as active structural acoustic control (ASAC), has been implemented using both feedback and feedforward control approaches. ${ }^{2-6}$ The control approach to be implemented mostly depends on the nature of the disturbance input, i.e., steady-state sinusoid, random, or transient. For applications in which the noise field is due to persistent inputs, the potential of the ASAC technique in conjunction with adaptive feedforward control approaches has been clearly demonstrated. ${ }^{4-6}$

The design of feedforward control systems involves the selection of the type, number, location, and size of the actuators and of the error sensors whose outputs are sought to be minimized. The traditional design approach in feedforward control is to select actuators and sensors based on some physical understanding of the behavior of the uncontrolled system. In general, this empirical methodology yields satisfactory results for ASAC when the error transducers are microphones placed in the acoustic field that directly observe the quantity to be minimized and when the excitation is a single sinusoid. However, this heuristic approach can easily result in an inefficient control system with an unnecessarily large number of control channels even in simple systems and is exacerbated when structural sensors are used. Wang et al $^{7}$ investigated the optimum location of actuators to minimize radiation from panels with microphones used as error transducers. It is demonstrated in this work that for singlefrequency excitation, both on and off resonance, the optimally located actuators achieved a far better global reduction of sound than actuators whose position are chosen only upon some physical considerations.

As a result of rapid advances in specialized actuator and sensor materials, today research thrust is toward developing smart or adaptive systems with actuators and sensors being an integral part of the structure. ${ }^{8}$ The typical transducer to be embedded in a structure will be distributed in nature. In particular, induced strain piezoelectric (PZT) ceramics, ${ }^{9,10}$ and shape memory alloys ${ }^{11}$ as actuators, and polyvinylidene fluoride (PVDF) films ${ }^{12,13}$ and fiber optics ${ }^{14}$ as sensors have shown potential for ASAC applications. The design of control systems of structures with integrated transducers will be even more critical since these error sensors will not directly measure acoustic pressure which is the quantity to be reduced and not all structural motion is well coupled to the radiation field. Clark et al. ${ }^{15}$ extended the work of Wang ${ }^{7}$ to optimize the location of piezoelectric actuators and both the location and size of PVDF strain sensors on a simply supported plate excited harmonically. Analytical and experimental results showed that a simple single optimally located PZT actuator/PVDF sensor pair rivaled the sound reduction achieved with three arbitrarily located PZT actuators and three error microphones.

These formal optimization approaches ${ }^{7,15}$ clearly dem- 
onstrate that optimally located actuators and sensors can have a profound impact on the performance of the active control system. Even more important is the fact that significant levels of attenuation can be obtained with far less numbers of properly located transducers, thus reducing the dimensionality and complexity of the controller. Unfortunately, these direct optimization techniques require the evaluation of the radiated pressure at each step of the minimization process. The acoustic prediction that will certainly be carried out numerically for real structures is a computationally intensive analysis. Thus, these design optimization techniques cannot be realistically implemented to complex structures and disturbances in the present form because of computational time aspects.

In this paper, a new efficient design formulation for feedforward ASAC systems is proposed. The actuators and sensors are designed such that the controlled structure will respond with a set of poor radiating modes. To this end, the formulation takes advantage of recent work that demonstrated that an active feedforward-controlled structure will respond with a new set of eigenproperties whether vibration or radiation is being attenuated. ${ }^{16,17}$ It was demonstrated that for radiation control the controlled eigenfunctions were all nonvolumetric or inefficient (weak) radiators. ${ }^{17,18}$ The controlled resonant frequencies and associated eigenfunctions are a function of the selected control actuators and error sensors and are independent of the disturbance input. Thus, the design approach is based on finding the actuators and sensors configuration such that the controlled structure will respond with the weakest set of modal radiators. These weak radiators are defined here as the eigenfunctions with the lowest radiation efficiency and are obtained by solving an eigenvalue problem. The formulation is valid for both narrowband and broadband excitation inputs. The design formulation is demonstrated on a simple supported plate excited with white noise.

\section{SYSTEM RESPONSE}

The structure is assumed linear and subjected to a stationary disturbance input. Thus the analysis is carried out in the frequency domain by simply taking the Fourier transform of any time-dependent variable. For the sake of clarity, the formulation will be presented for a planar radiator and a single-input, single-output (SISO) control system. However, there is no loss of generality in the design methodology proposed here. A typical SISO feedforward-control arrangement is shown in Fig. 1. In feedforward control the undesirable response of a system due to the "primary" disturbance input is reduced by applying a "secondary" control input. The control input is obtained by feeding a reference signal into the compensator, $G(\omega)$. The compensator is designed such that the output from an error sensor is minimized. In feedforward-control approaches, the reference signal should be "coherent" to the disturbance input signal, and it is assumed here that it is directly obtained by tapping the disturbance input.

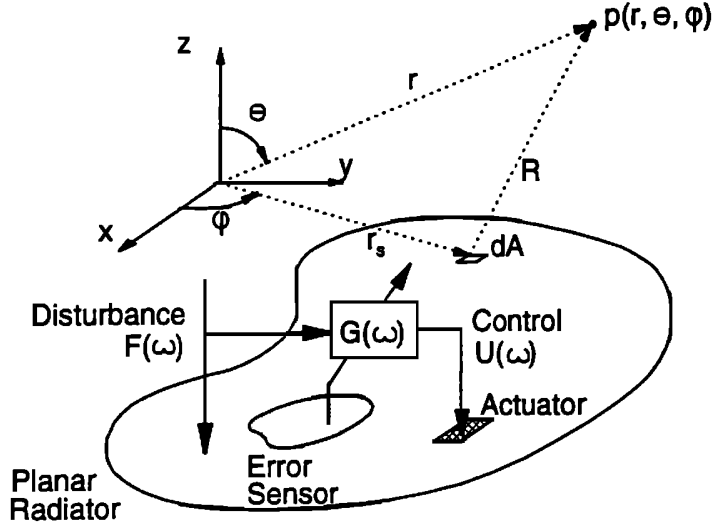

FIG. 1. General arrangement of a SISO feedforward ASAC system.

\section{A. Structural response}

The structural response $w(x, y, \omega)$ can be expressed as a linear combination of the modes as

$$
w(x, y, \omega)=\sum_{n=1}^{N} q_{n}(\omega) \phi_{n}(x, y),
$$

where $q_{n}(\omega)$ is the $n$th generalized modal coordinate, $\phi_{n}(x, y)$ is the $n$th eigenfunction, and $N$ is the number of modes included in the response. The modal displacement can be written as ${ }^{16}$

$$
q_{n}(\omega)=\left[f_{n} F(\omega)+u_{n} U(\omega)\right] H_{n}(\omega),
$$

where $f_{n}$ is the $n$th modal disturbance force, $F(\omega)$ is the Fourier transform of the disturbance input, $u_{n}$ is the $n$th unit modal control force, $U(\omega)$ is the Fourier transform of the control input, and $H(\omega)$ is the $n$th modal frequency response function, given as

$$
H_{n}(\omega)=\frac{1}{\omega_{n}^{2}-\omega^{2}+j 2 \zeta_{n} \omega_{n} \omega},
$$

where $\omega_{n}$ and $\zeta_{n}$ are the $n$th natural frequency and modal damping ratio, respectively, and $j$ is the imaginary number. Equation (2) assumes that the modes have been normalized with respect to the mass distribution.

The optimum frequency content of the control input stems from minimizing a cost function, which is the meansquare value of a structural response monitored by an error sensor. Since the goal of this work is to design what is termed as adaptive structural systems, actuators and sensors contained within the structure will be the only transducers considered in this study. Then, the Fourier transform of the error sensor output can also be represented as the sum of the linear contribution of each mode as follows: ${ }^{7}$

$$
e(\omega)=\sum_{n=1}^{N} q_{n}(\omega) \xi_{n},
$$

where $\xi_{n}$ is the $n$th modal error component which is a function of the characteristics of the error transducer, i.e., discrete or distributed.

The modal error components $\xi_{n}$ and the unit modal control forces $u_{n}$ are a measure of the relative observability and 
controllability of the modes by the error sensor and control actuator, respectively. By minimizing the cost function with respect to the control input, it can be shown that the spectrum content of the control input is given by ${ }^{16,19}$

$$
U(\omega)=G(\omega) F(\omega)=-\frac{\sum_{n=1}^{N} \xi_{n} f_{n} H_{n}(\omega)}{\sum_{n=1}^{N} \xi_{n} u_{n} H_{n}(\omega)} F(\omega),
$$

where $G(\omega)$ is the compensator that relates the control input $U(\omega)$ to the disturbance input $F(\omega)$. For a nonminimum phase control system the optimum compensator $G(\omega)$ is not implementable for broadband disturbances. For single and multiple frequency disturbances, the optimum compensator is always realizable. As discussed later, the design approach presented here will yield a control-error path that is minimum phase and thus the design formulation is applicable to any type of input. The controlled response can now be computed by solving $\mathrm{Eq}$. (5) for the control input $U(\omega)$ and replacing it into Eq. (2) and this into Eq. (1). The uncontrolled response can be computed by simply setting $U(\omega)$ to zero in Eq. (2). In this SISO control configuration, the error output is theoretically driven to zero by the control input at all frequencies.

\section{B. Acoustic response}

The far-field pressure radiated by a harmonically vibrating planar structure can be computed from the structural response by using the Raleigh integral ${ }^{20}$ as follows:

$$
p(\vec{r}, \omega)=\frac{i \omega \rho_{0}}{2 \pi} \int_{A} v\left(x_{s}, y_{s}\right) \frac{e^{-j k R}}{R} d A,
$$

where, as shown in Fig. $1, \vec{r}=(r, \theta, \varphi)$ is the polar coordinate of the observation point in the acoustic field; $r_{s}=\left(x_{s}, y_{s}\right)$, where $x_{s}$ and $y_{s}$ are the coordinates of the elemental surface $d A$ having normal velocity $v\left(x_{s}, y_{s}\right) ; A$ is the area of the radiator; $R$ is the magnitude of the distance from the elementary source and the observation point; $\rho_{0}$ is the fluid density; and $k$ is the acoustic wave number given as $k=\omega / c$, where $c$ is the speed of sound in the medium. Here, it is assumed that the acoustic medium is air and thus no feedback of the fluid motion into the structure takes place.

Again the radiated pressure can be expressed as a linear contribution of the modes as

$$
p(\vec{r}, \omega)=\sum_{n=1}^{N} q_{n}(\omega) p_{n}(\vec{r}, \omega),
$$

where

$$
p_{n}(\vec{r}, \omega)=\frac{i \omega \rho_{0}}{2 \pi} \int_{A} i \omega \phi_{n}\left(x_{s}, y_{s}\right) \frac{e^{-j k R}}{R} d A
$$

is the radiated pressure distribution given by the $n$th mode with surface velocity $i \omega \phi_{n}(x, y)$. The controlled acoustic sound field can be computed by replacing Eq. (2) into (7).

It should be noted that typically the computation of the radiated pressure for a complex structure will be performed numerically by finite/boundary element codes. ${ }^{21}$ This analysis is a computationally intensive process, and therefore any efficient controller design approach should minimize the number of acoustic evaluations. The following work is directed toward achieving this goal.

\section{CONTROLLED SYSTEM EIGENPROPERTIES}

The previous analysis provides the tools to compute the controlled structural and acoustic responses. However, it does not give any insight into the control mechanisms. Since the design approach proposed here is founded on the understanding of these mechanisms, the main aspects of the dynamic behavior of feedforward-controlled structures will be described in the sequel, while a full detailed description can be found in Ref. 16. The traditional view of feedforwardcontrolled systems is of "active cancellation" where the modes of the structure excited by the "primary" disturbance input are canceled by the "secondary" control input of appropriate magnitude and phase driving the same structural modes. This view arises from the fact that the system response can be interpreted as the superposition of the disturbance and control responses as suggested by Eq. (2). However, recent work has shown that the feedforward-controlled system responds effectively with a new set of eigenfunctions and eigenvalues to the disturbance input. ${ }^{16}$

The eigenproperties of the feedforward controlled system are governed by the characteristics of the controller $G(\omega)$ in Eq. (5). Because of the constraint imposed on the structure by driving the error signal to zero at all frequencies, it can be shown that the controller reduces the dynamic degrees of freedom of the system by 1 , and thus the controlled system has $(N-1)$ new eigenvalues and associated eigenfunctions. The undamped controlled system eigenvalues, $\lambda_{l}$, are computed such that the following equation is satisfied:

$$
\sum_{n=1}^{N} \xi_{n} u_{n} \prod_{\substack{m=1 \\ m \neq n}}^{N}\left(\mu_{m}-\lambda_{l}\right)=0, \quad l=1, \ldots, N-1,
$$

where $\mu_{m}=\left(\omega_{m}\right)^{2}$ is the $m$ th uncontrolled system eigenvalue. This implies that if the structure is excited at a frequency near the control system pole frequency, $f_{l}=\left(\lambda_{l}\right)^{1 / 2} / 2 \pi$, the controlled structural response will be very large (or unbounded for an undamped system, $\zeta_{n}=0$ ). This behavior takes place even though the error sensor output vanished at all frequencies.

The controlled system eigenfunction $\phi_{l}(x, y)$ associated to the eigenvalue $\lambda_{l}$ is easily computed once the controlled system eigenvalue has been determined. They can be obtained as a linear combination of the uncontrolled modes. That is,

$$
\phi_{l}(x, y)=\sum_{n=1}^{N} \Gamma_{l n} \phi_{n}(x, y)
$$

and the expansion coefficients are

$$
\Gamma_{l n}=C_{l} u_{n} /\left(\lambda_{l}-\mu_{n}\right), \quad n=1, \ldots, N,
$$

where $C_{l}$ is a constant that is included since the controlled mode shapes are arbitrary to a constant multiplier, and it can be computed such that $\left[\left(\Gamma_{l 1}\right)^{2}+\left(\Gamma_{l 2}\right)^{2}+\cdots+\left(\Gamma_{l N}\right)^{2}\right]=1$. 
The eigenproperties of the controlled system given by Eqs. (9)-(11) are a function of the control input, through the unit modal control forces $u_{n}$, and of the error variable, through the modal error components $\xi_{n}$. An important aspect of the eigenproperties are that they are independent of the disturbance input. This implies that once the control actuator and error sensor are selected the eigenstructure of the controlled system is completely determined independently of the nature of the disturbance input including frequency content, location and distribution.

\section{DESIGN APPROACH}

The main goal in ASAC is for the control system to render a controlled response that poorly couples with the acoustic medium, thus resulting in minimum radiated sound power. This objective can be accomplished if two conditions are met.

(i) The resonant frequencies of the controlled structure must lay away from the dominant part of the disturbance input spectrum. In other words, the controlled system resonances should be detuned from the excitation input.

(ii) More important, the controlled or residual structural response should be a linear contribution of weak radiating modes.

As mentioned in the previous section, the controlled system has new resonant frequencies and associated eigenfunctions that are only a function of the selected actuator and sensor. Thus, this concept can be merged with the above conditions to yield an efficient design approach. The design formulation proposed here can be stated as to find the optimum actuator and sensor configuration that yields a controlled structure with eigenproperties that satisfied the above two conditions.

The first step in the proposed design formulation is to find the desired set of controlled system eigenfunctions with which the controlled structure should respond. The sought eigenfunctions should be weak modal radiator and this can be mathematically formulated by requiring the desired eigenfunctions to have the lowest radiation efficiency possible. Since the controlled eigenfunctions are given as a linear combination of the uncontrolled eigenfunctions, this implies a search for the desired expansion coefficients $\left(\Gamma_{l n}\right)_{d}$ in order to achieve this objective.

To compute the radiation efficiency, the ratio of the radiated power to the average mean square velocity of the radiating surface is required. The radiated pressure due to the lth controlled eigenfunction driven harmonically at frequency $\omega$ can be computed using the modal surface velocity distribution $v(x, y)=i \omega \phi_{l}(x, y)$ substituted into the Rayleigh integral, ${ }^{20} \mathrm{Eq}$. (6). Considering Eq. (10), the controlled modal pressure distribution is given in terms of the unknown desired coefficients, $\left(\Gamma_{l n}\right)_{d}$, and of the uncontrolled modal pressure distribution, $p_{n}(\vec{r}, \omega)$, as

$$
p_{l}(\vec{r}, \omega)=\sum_{n=1}^{N}\left(\Gamma_{l n}\right)_{d} p_{n}(\vec{r}, \omega)
$$

The radiated sound power at frequency $\omega$ due to the $l$ th controlled mode over the region $D$ in the acoustic field is computed by integrating the acoustic time average intensity as

$$
\Pi_{l}(\omega)=\iint_{D} \frac{\left|p_{l}\right|^{2}}{\rho_{0} c} d D
$$

and replacing Eq. (12) into (13) gives

$$
\begin{aligned}
\Pi_{l}(\omega) & =\sum_{n=1}^{N} \sum_{m=1}^{N}\left(\Gamma_{l n}\right)_{d}\left(\Gamma_{l m}\right)_{d} \Pi_{n m}(\omega) \\
& =\left\{\Gamma_{l}\right\}_{d}^{T}[\Pi(\omega)]\left\{\Gamma_{l}\right\}_{d},
\end{aligned}
$$

where the elements of matrix $[\Pi(\omega)]$ are

$$
\Pi_{n m}(\omega)=\iint_{D} \frac{p_{n} p_{m}^{*}}{\rho_{0} c} d D
$$

the desired expansion vector is $\left\{\Gamma_{l}\right\}_{d}=\left\{\left(\Gamma_{l 1}\right)_{d}, \ldots,\left(\Gamma_{l N}\right)_{d}\right\}^{T}$, and the superscript $T$ denotes the vector transpose.

Matrix $[\Pi(\omega)]$ is symmetric and positive definite because the radiated power is always positive for a nontrivial vector $\left\{\Gamma_{l}\right\}_{d}$. The term $\Pi_{n n}(\omega)$ represents the radiated power by the $n$th uncontrolled eigenfunction, while the term $\Pi_{n m}(\omega)$ represents the radiated power due to the acoustic coupling between the $n$th and $m$ th uncontrolled eigenfunctions. If the cross term $\Pi_{n m}(\omega)$ vanishes, the modes are said to be acoustically uncoupled. The important implication is that the radiation due to the $n$th mode can not be used to destructively interfere with the radiation due to the $m$ th mode such that the average radiated power over the region $D$ is reduced.

The average mean-square velocity for the controlled mode oscillating at frequency $\omega$ can be easily computed as

$$
\left|v_{l}(\omega)\right|^{2}=\frac{\omega^{2}}{2 A} \iint_{A} \phi_{l}^{2}\left(x_{s}, y_{s}\right) d A
$$

Again, it is straightforward to show that the average meansquare velocity becomes

$$
\left|v_{l}(\omega)\right|^{2}=\left\{\Gamma_{l}\right\}_{d}^{T}[V(\omega)]\left\{\Gamma_{l}\right\}_{d},
$$

where matrix $[V(\omega)]$ is also symmetric and positive definite, and its elements are

$$
V_{n m}(\omega)=\frac{\omega^{2}}{2 A} \iint_{A} \phi_{n}\left(x_{s}, y_{s}\right) \phi_{m}\left(x_{s}, y_{s}\right) d A
$$

Finally the radiation efficiency of the $l$ th controlled eigenfunction is defined as ${ }^{22}$

$$
S_{l}(\omega)=\frac{1}{A \rho_{0} c} \frac{\Pi_{l}(\omega)}{\left|v_{l}(\omega)\right|^{2}}=\frac{1}{A \rho_{0} c} \frac{\left\{\Gamma_{l}\right\}^{T}[\Pi(\omega)]\left\{\Gamma_{l}\right\}}{\left\{\Gamma_{l}\right\}^{T}[V(\omega)]\left\{\Gamma_{l}\right\}} .
$$

The desired expansion coefficients $\left(\Gamma_{l n}\right)_{d}$ that yield the lowest radiation efficiency for the controlled modes are obtained by minimizing $S_{l}(\omega)$ in Eq. (19) with respect to the coefficients $\left(\Gamma_{l n}\right)_{d}$ with the constraint $\left(\left\{\Gamma_{l}\right\}_{d}\right)^{T}\left\{\Gamma_{l}\right\}_{d}=1$. This constrained minimization problem can be efficiently 
solved by recognizing that the stationary values of $S_{l}(\omega)$ can be obtained by solving the eigenvalue problem

$$
\left\{[\Pi(\omega)]-S_{l}[V(\omega)] A \rho_{0} c\right\}\left\{\Gamma_{l}\right\}_{d}=\{0\}, \quad l=1, \ldots, N .
$$

Because of the symmetry and positive-definite properties of the matrices, the $N$ eigenvalues $S_{l}$ are all real and positive and they are ordered such that $S_{1}<S_{2}<\cdots<S_{N}$. The eigenvalue $S_{l}$ represents the radiation resistance at frequency $\omega$ of the $l$ th desired controlled eigenfunction that is given as a linear combination of the uncontrolled modes by the coefficients of the eigenvector $\left\{\Gamma_{l}\right\}_{d}$. Because the SISO control system considered here has $(N-1)$ eigenfunctions, the optimum set of controlled eigenfunctions is that with the $(N-1)$ lowest radiation efficiency. Thus the desired expansion coefficients $\left(\Gamma_{l n}\right)_{d}$ are the first $(N-1)$ eigenvectors in Eq. (20). These eigenfunctions form a set of independent basis functions for the controlled system.

The desired eigenfunctions found above are optimum in the sense that they have the lowest radiation efficiency at the single frequency $\omega$. For broadband excitation, the controlled eigenfunctions should have low radiation efficiency in the frequency range of the excitation input. This can be accomplished by solving for the minimum of the weighted radiation efficiency:

$$
S_{l}(\omega)=\frac{1}{A \rho_{0} c} \frac{\left\{\Gamma_{l}\right\}_{d}^{T} \Sigma_{i=1}^{L} B_{i}\left[\Pi\left(\omega_{i}\right)\right]\left\{\Gamma_{l}\right\}_{d}}{\left\{\Gamma_{l}\right\}_{d}^{T} \Sigma_{i=1}^{L} B_{i}\left[V\left(\omega_{i}\right)\right]\left\{\Gamma_{l}\right\}_{d}},
$$

where $B_{i}$ is a weighting constant, and matrices $\left[\Pi\left(\omega_{i}\right)\right]$ and [V $\left.\left(\omega_{i}\right)\right]$ are computed at $L$ frequencies $\omega_{i}$. The eigenproblem of Eq. (21) will then yield a set of optimum eigenfunctions that have low radiation efficiency, in some weighted sense, at the $L$ frequencies $\omega_{i}$. Then, the computation of the uncontrolled modes radiated pressure is required at $L$ frequencies. This analysis, in particular if numerical techniques are used, is a computational intensive process and care should be exercised in selecting both the number and values of the frequencies $\omega_{i}$. Sensitivity analysis of the acoustic pressure with respect to the frequency should be useful here. $^{23,24}$

It should be mentioned that Cunefare ${ }^{25}$ developed a similar eigenvalue formulation for obtaining the optimum velocity distribution on a finite beam that minimizes the radiation efficiency of the beam response at a single frequency. It was suggested in this work that the optimum velocity response could be used as the objective function for the design of active control systems. Though similar in concept, the proposed formulation differs markedly from Cunefare's work in a fundamental aspect. Here the formulation searches for controlled eigenfunctions that are weak radiators and that are independent of the frequency.

\section{A. Optimum modal parameters}

The expansion coefficients $\Gamma_{l n}$ are a function of the unit modal control forces $u_{n}$, the uncontrolled eigenvalues $\mu_{n}$, and the controlled eigenvalues $\lambda_{l}$, as depicted by Eq. (11). Thus the unit modal control forces and the controlled eigenvalues can now be determined so they yield the desired expansion coefficients found from the solution of the above eigenproblem. However, the number of expansion coefficients to match is $N \times(N-1)$ while there are $2(N-1)$ design variables, i.e., $(N-1)$ relative modal control forces and $(N-1)$ controlled eigenvalues. Therefore, the desired expansion coefficients can be achieved only in some least-square sense. The controlled eigenvalues $\lambda_{l}$ and modal control forces $u_{n}$ can then be obtained by solving the following least-square constrained minimization problem:

Minimize

$$
F\left(u_{n}, \lambda_{l}\right)=\sum_{l=1}^{N-1} \sum_{n=1}^{N}\left|\left(\Gamma_{l n}\right)_{d}-c_{l} \frac{u_{n}}{\lambda_{l}-\mu_{n}}\right|^{2}
$$

such that

$$
\sum_{n=1}^{N} u_{n}^{2}=1, \quad\left(\lambda_{l}\right)_{\text {lower }} \leqslant\left(\lambda_{l}\right) \leqslant\left(\lambda_{l}\right)_{\text {upper }} .
$$

The equality constraint represents the normalization of the modal control forces since the relative controllability of the modes is the only relevant information. The upper and lower limits of the inequality constraints on the values for the controlled system eigenvalues $\lambda_{l}$ are selected based on the characteristic of the spectrum content of the disturbance input. For example, if the disturbance input consists of multiple sinusoids, the controlled eigenvalues are selected such that they are not coincident with any of the excitation sinusoids. It is also important to remark that the controlled eigenvalues $\lambda_{l}$ are restricted to have real positive values. The implication of this constraint is that the optimum compensator $G(\omega)$ is physically realizable. The solution of the minimization problem can be carried out efficiently by a number of optimization routines and yields the unit modal control forces $u_{n}$ and the controlled eigenvalues $\lambda_{l}$.

Recent work $^{17}$ has shown that, given $u_{n}$ and $\lambda_{l}$, the error modal components $\xi_{n}$ can be computed from the characteristic equation of the controlled system in Eq. (9). Replacing the optimum controlled eigenvalues $\lambda_{l}$ and unit modal control forces $u_{n}$ found from the solution of the above optimization problem into Eq. (9) gives ${ }^{17}$

$$
\begin{gathered}
{\left[\begin{array}{cccc}
A_{11} & A_{12} & \cdots & A_{1 N} \\
A_{21} & A_{22} & \cdots & A_{2 N} \\
\vdots & & \ddots & \vdots \\
A_{(N-1) 1} & A_{(N-1) 2} & \cdots & A_{(N-1) N}
\end{array}\right]\left\{\begin{array}{c}
\xi_{1} \\
\xi_{2} \\
\vdots \\
\xi_{N}
\end{array}\right\}=\left\{\begin{array}{c}
0 \\
0 \\
\vdots \\
0
\end{array}\right\},} \\
\text { with } \quad A_{l n}=u_{n} \prod_{\substack{m=1 \\
m \neq n}}^{N}\left(\mu_{n}-\lambda_{l}\right) .
\end{gathered}
$$

The homogeneous linear system of equations is such that the only relevant information is the relative observability of the uncontrolled modes by the error sensor. Thus assuming the $N$ th mode is observable, then by setting $\xi_{N}$ to 1 , the modal error components can be obtained by solving the reduced linear system of equations 


$$
\begin{gathered}
{\left[\begin{array}{cccc}
A_{11} & A_{12} & \cdots & A_{1(N-1)} \\
A_{21} & A_{22} & \cdots & A_{2(N-1)} \\
\vdots & & \ddots & \vdots \\
A_{(N-1) 1} & A_{(N-1) 2} & \cdots & A_{(N-1)(N-1)}
\end{array}\right]\left\{\begin{array}{c}
\xi_{1} \\
\xi_{2} \\
\vdots \\
\xi_{N-1}
\end{array}\right\}} \\
=-\left\{\begin{array}{c}
A_{1 N} \\
A_{2 N} \\
\vdots \\
A_{(N-1) N}
\end{array}\right\}
\end{gathered}
$$

The optimal modal error $\xi_{n}$ and control $u_{n}$ components define completely the control system configuration in the modal domain. The design of the control system in the modal domain as carried out in this formulation offers a number of advantages. The performance of the controller can be investigated with the modal parameters alone before the transducers are selected. Control issues such as number of control channels for a required reduction can be addressed effectively. This approach also allows for the investigation of different kinds of actuators and sensors with minimum additional computational effort.

This modal information needs to be transformed into physical transducers that can then be implemented on the structure. The type of actuator and sensor, i.e., discrete or distributed, to be implemented is an application-dependent problem and beyond the main focus of the proposed design approach. For the sake of completeness, the implementation of physical strain-induced transducers is, however, illustrated in the numerical section.

\section{NUMERICAL EXAMPLE}

The applicability of the design formulation presented here is demonstrated for an uniform simple supported plate. This structure was selected because analytical solution of both the structural response and the radiated sound field are available. The plate is assumed made of steel with density $\rho_{s}=7833 \mathrm{~N} \cdot \mathrm{s}^{2} / \mathrm{m}^{4}$, Young's modulus $E=2.0 \times 10^{11} \mathrm{~N} / \mathrm{m}$, Poison's ratio $\nu=0.3$, thickness $h=0.002 \mathrm{~m}$, and dimensions $L_{x}=0.38 \mathrm{~m}$ and $L_{y}=0.3 \mathrm{~m}$. The spectrum of the disturbance excitation is assumed to be white noise in the [0-800] frequency band. To compute the response of the system, it is assumed a modal damping ratio of $1.0 \%$ in all modes $\left(\zeta_{n}=0.01\right)$, and the number of modes included in the analysis is $N=8$. Since the proposed design is based on the modal representation of the system's response, the $n$th natural frequency an eigenfunction of the plate is

$$
\begin{aligned}
& \omega_{n}=\sqrt{D_{p} / \rho_{s} h}\left(\gamma_{x}^{2}+\gamma_{y}^{2}\right), \\
& \phi_{n}(x, y)=K \sin \left(\gamma_{x} x\right) \sin \left(\gamma_{y} y\right),
\end{aligned}
$$

where $\gamma_{x}=\pi n_{x} / L_{x}, \gamma_{y}=\pi n_{y} / L_{y}, D_{p}=E h^{3} / 12\left(1-\nu^{2}\right)$ is the flexural rigidity, $\left(n_{x}, n_{y}\right)$ are the modal indices traditionally used for rectangular panels that are associated to the $n$ index used in the theoretical analysis, $n \rightarrow\left(n_{x}, n_{y}\right)$, and $K=\left(4 / L_{x} L_{y} h \rho_{s}\right)^{1 / 2}$.

The plate is assumed baffled to eliminate the acoustic interaction between the back and front radiation and facilitate the analytical predictions. The radiated far-field pressure due to each of the plate modes was first computed by solving the Rayleigh integral in Eq. (8). Assuming the observation point to be in the far field $\left(k L_{x} \gg 1\right.$ and $\left.k L_{y} \gg 1\right)$, Eq. (8) has a closed-form solution given by ${ }^{22}$

$$
\begin{aligned}
p_{n}(\vec{r}, \omega)= & \frac{-\omega^{2} \rho_{0} L_{x} L_{y} K}{2 \pi^{3} n_{x} n_{y}} \frac{e^{-j k r}}{r}\left(\frac{(-1)^{n_{x}} e^{-j k \alpha}-1}{\left(\alpha / n_{x} \pi\right)^{2}-1}\right) \\
& \times\left(\frac{(-1)^{n_{y}} e^{-j k \beta}-1}{\left(\beta / n_{y} \pi\right)^{2}-1}\right),
\end{aligned}
$$

where

$$
\alpha=k L_{x} \sin \theta \cos \varphi, \quad \beta=k L_{y} \sin \theta \sin \varphi .
$$

The uncontrolled modal far-field pressure distribution in Eq. (27) can now be used to compute the auto- and crossmodal radiation power terms in Eq. (15). The aim in this control example is to attenuate the total radiated power. Thus the integration of the local intensity is carried out over one half hemisphere, which for convenience is computed numerically.

The modes of a simply supported plate can be collected in four groups which are the odd-odd, even-odd, odd-even, and even-even modes. Solving Eq. (15) shows that the cross-radiation power terms between modes from different groups vanish. This implies that the modes in a group are acoustically uncoupled from the modes of the other groups. Conversely, the only acoustic coupling takes place between modes of the same group. For example, the $(1,1),(3,1)$, and $(1,3)$ odd-odd modes have nonzero off-diagonal elements. This implies attenuation of sound produced by these modes can be achieved by these modes interacting with each other. In other words the cross-radiated power would balance the direct radiated power. This is the essence of ASAC where sound attenuation can be achieved by restructuring the acoustically efficient modes rather than controlling each one of them. On the other hand, the $(1,2)$ and $(3,2)$ odd-even modes are not acoustically coupled with the odd-odd modes. Thus the radiation over a half-hemisphere due to these modes cannot be canceled by the odd-odd modes. It is evident that for the simply supported plate problem the oddodd, even-odd, odd-even, and even-even modes should be controlled independently. Thus, a control system design should start by careful identification of not only the strongly radiating modes but also of the acoustic coupling.

The applicability of the design formulation is demonstrated for controlling the radiation due to the $(1,1),(3,1)$, and $(1,3)$ modes with a SISO controller. To this end, it is assumed that these are the only modes excited by both the disturbance and control inputs and observed by the error sensor. This can be expressed mathematically by setting the modal parameters $f_{n}, u_{n}$, and $\xi_{n}$ to zero for all modes except the odd-odd ones.

\section{A. Desired controlled eigenfunctions}

The first step is the design process is to find the desired controlled system eigenfunctions. The single frequency of $300 \mathrm{~Hz}$ was selected to compute the matrices $[\Pi(\omega)]$ and $[V(\omega)]$ to form the eigenvalue problem of Eq. (20). The solution of the eigenvalue problem yielded three eigenvalues 
TABLE I. Radiation efficiency and expansion coefficients of desired eigenfunctions.

\begin{tabular}{ccc}
\hline \hline \multicolumn{3}{c}{ Radiation efficiency, $S_{l} \times 10^{-3}$} \\
\hline \multicolumn{3}{c}{0.239} \\
\\
& Desired coefficients, $\left\{\Gamma_{l}\right\}_{d}$ \\
\hline$(1,1)$ & -0.367 & 0.128 \\
$(3,1)$ & 0.361 & -0.893 \\
$(1,3)$ & 0.857 & 0.431 \\
\hline
\end{tabular}

and associated eigenvectors. Since the SISO controller reduces the number of dynamic degrees of freedom by 1 , the desired expansion coefficients $\left(\Gamma_{l n}\right)_{d}$ are the first two eigenvectors. The radiation efficiency at $300 \mathrm{~Hz}$, eigenvalues $S_{l}$, and associated expansion eigenvectors $\left\{\Gamma_{l}\right\}_{d}$ of the desired controlled eigenfunctions are given in Table $\mathrm{I}$.

\section{B. Optimum modal parameters}

The optimum modal parameters, i.e., modal control and error components, can now be computed. The next step is to find the unit modal control forces $u_{n}$ and controlled eigenvalues $\lambda_{n}$ that yield the desired expansion coefficients of Table I. This is achieved by solving the minimization problem in Eqs. (22) and (23) where the controlled eigenvalues $\lambda_{l}$ are not constrained as in Eq. (23) because the spectrum of the excitation input is white noise. The minimization process was carried out by using the optimization IMSL routine DUNLSF (nonlinear least squares problems), which yielded the optimum unit modal control forces $u_{n}$ shown in Table II and two controlled resonant frequencies at 399 and $700 \mathrm{~Hz}$ as shown in Table III. These values yielded the expansion coefficients shown in columns 2 and 3 of Table III. These coefficients are almost identical to the desired coefficients obtained from the solution of the eigenvalue problem in Eq. (20) and presented in Table I. The modal control forces and controlled eigenvalues can now be used in the linear system of equations in Eq. (26) to solve for the modal error components $\xi_{n}$; they are given in Table III.

The resulting modal parameters listed in Table II define completely the control system. The controller has modified the eigenstructure of the system in such a way that the controlled structure will respond with two weak radiating modes. To illustrate this fact, the radiation efficiency for the three odd-odd uncontrolled eigenfunctions and the two new controlled eigenfunctions was computed and they are plotted in Fig. 2. This figure shows clearly that the controlled modes have substantially lower radiation efficiency than the uncontrolled modes. It is also very interesting to note that, by

TABLE II. Modal control and error components.

\begin{tabular}{rrc}
\hline \hline & \multicolumn{2}{c}{ Modal parameters } \\
\cline { 2 - 3 }$\left(n_{x}, n_{y}\right)$ & $u_{n}$ & $\xi_{n}$ \\
\hline$(1,1)$ & -0.534 & 0.924 \\
$(3,1)$ & 0.479 & 0.329 \\
$(1,3)$ & 0.697 & 0.192 \\
\hline \hline
\end{tabular}

TABLE III. Controlled system eigenproperties.

\begin{tabular}{ccc}
\hline \hline \multicolumn{3}{c}{ Resonant frequency $f_{l}=\left(\lambda_{l}\right)^{1 / 2} / 2 \pi(\mathrm{Hz})$} \\
$f_{1}=399$ & $f_{2}=700$ \\
$\left(n_{x}, n_{y}\right)$ & Expansion coefficients $\Gamma_{l n}$ & $\Gamma_{2 n}$ \\
\hline$(1,1)$ & $\Gamma_{1 n}$ & -0.314 \\
$(3,1)$ & 0.224 & 0.371 \\
$(1,3)$ & -0.877 & 0.874 \\
\hline
\end{tabular}

numerical integration, the controlled eigenfunctions are nonvolumetric. Since the response is a linear expansion of the modes, this implies that the net volume displaced by the controlled plate is zero at all frequencies. This same phenomenon was observed by the authors ${ }^{18}$ in studying the dynamic behavior of feedforward-controlled systems using microphones in the far field as error sensors.

The sensitivity of the controlled eigenfunctions to the selected frequency used in Eq. (20) is an important issue. Solving the eigenproblem in Eq. (20) for different values of the frequency within the excitation band showed that the desired expansion coefficients did not vary substantially. Even though this is not a general conclusion, this suggests that in the proposed design approach the solution of the modal acoustic field could be efficiently carried out at a small number of frequencies. Since this analysis is the most computational intensive process, the proposed design approach has clear computational benefits over direct optimization methods for complex structures and excitations.

\section{Control system performance}

The control system is completely defined in the modal domain in terms of the optimal modal parameters in Table II. The performance of the controlled structure can be investigated with these modal parameters alone before physical transducers are devised. The effectiveness of the control system was evaluated for a disturbance input consisting of a

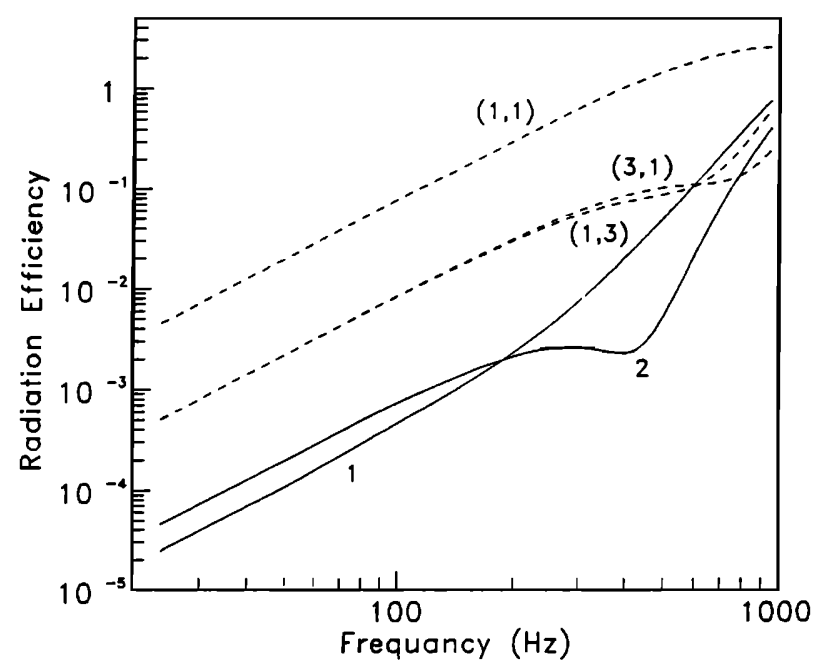

FIG. 2. Radiation efficiency of uncontrolled (----) and controlled ( - ) eigenfunctions. 


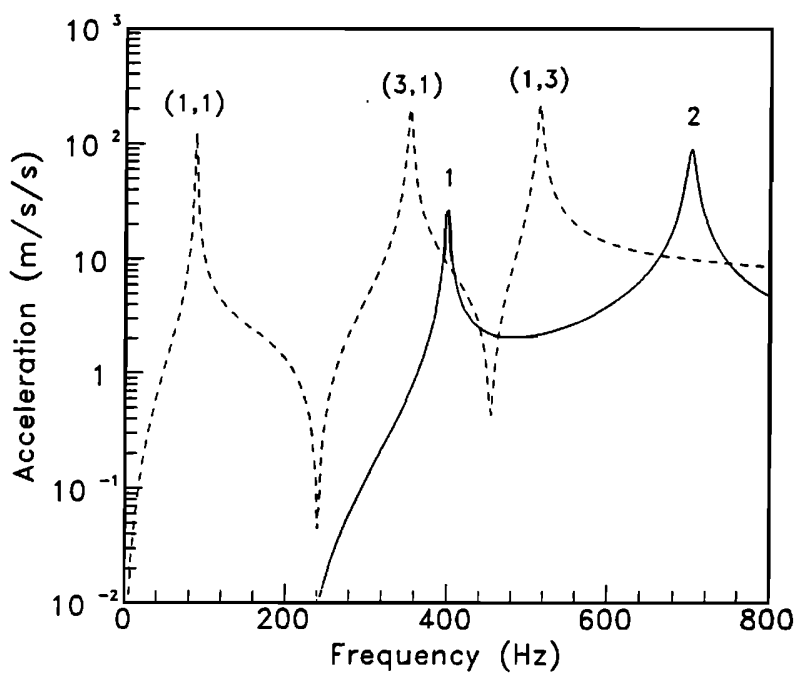

FIG. 3. Acceleration response at disturbance force location before (----) and after $(\longrightarrow$ ) control.

point force located at $x=0.5 L_{x}$ and $y=0.5 L_{y}$. To illustrate the dynamic behavior of the plate before and after control, the acceleration response of the plate at the disturbance location was computed. The amplitude is shown in Fig. 3 as a function of the frequency. The dashed line is the uncontrolled response and shows resonant peaks at the frequencies given in Eq. (27). On the other hand, when the control input is applied, the response shows resonance behavior at the two controlled resonant frequencies given in Table III. The vibration levels of the controlled structure are slightly lower than the uncontrolled system, i.e., amplitude of the second controlled mode is the same as the $(1,1)$ uncontrolled mode. Similarly, the before- and after-control far field pressure at $\vec{r}=\left(0^{\circ}, 0^{\circ}, 4.5 L_{x}\right)$ was also computed. The sound pressure level in decibels $(\mathrm{dB}$ re: $20 \mu \mathrm{Pa})$ is shown in Fig. 4 as a function of the frequency. This figure shows that the sound levels produced by the controlled structure are well below the level generated by the uncontrolled one. This is due to the low radiation efficiency of the controlled modes that re-

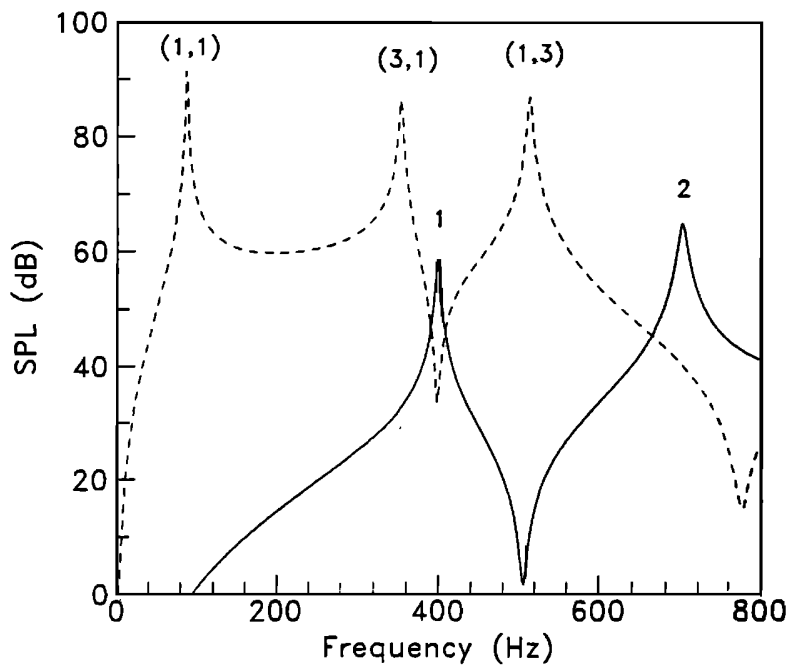

FIG. 4. Far-field pressure at $\vec{r}=\left(4.5 L_{x}, 0^{\circ}, 0^{\circ}\right)$ : uncontrolled (---.) and controlled $(\longrightarrow)$.

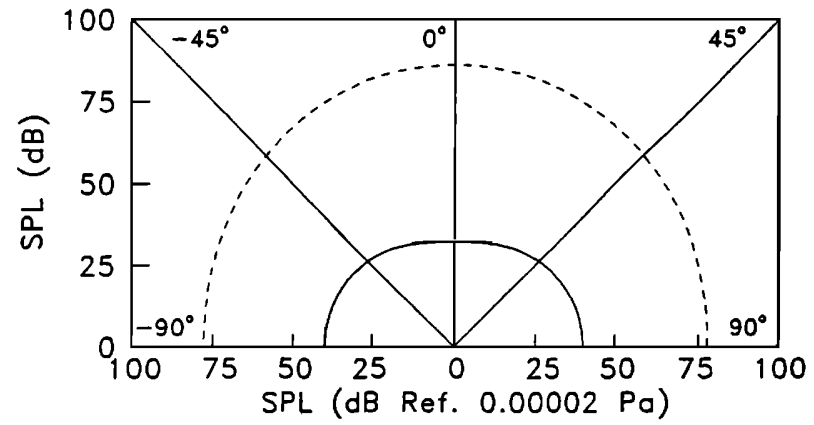

FIG. 5. Radiation directivity on the $x-z$ plane at $352 \mathrm{~Hz}$ : uncontrolled (----) and controlled ( $\longrightarrow$ ).

sults in a weak coupling of the controlled response with the acoustic medium. This phenomenon takes place in spite of the vibration levels being comparable to the uncontrolled response levels.

The radiation directivity in the horizontal $x-z$ planes was then computed at selected frequencies and they are shown in Figs. 5 and 6 . In all figures, the dashed and continuous lines represent the uncontrolled and controlled sound radiated pressure at a distance of $4.5 L_{x}$. Figure 5 shows the far-field radiation at $352 \mathrm{~Hz}$, corresponding to the resonance of the uncontrolled $(3,1)$ mode, which shows excellent global reduction. Similar behavior was observed for each of the uncontrolled resonant frequencies. More interesting is to investigate the radiated sound at the controlled system resonance frequencies. Figure 6 shows the far-field radiation at $399 \mathrm{~Hz}$, which is the second resonance of the controlled system. Inspection of this plot demonstrates that even though the response of the plate is very large (because of the resonance) the radiation is not increased significantly. Again this is because the controlled mode has very low radiation efficiency.

To evaluate the overall performance of the control configuration, the sound pressure spectrum was integrated over the bandwidth from 0 to $800 \mathrm{~Hz}$. The integrated spectrum is used to plot the overall radiation directivity in the $x-z$ plane. Total radiation directivities before and after control were computed for the $x-z$ planes and are shown in Fig. 7, respectively. These plots show that an average global sound pressure level reduction of $15 \mathrm{~dB}$ is obtained over the whole spectrum, thus attesting to the effectiveness of the design approach presented here.

The process of designing a structural actuator and sensor

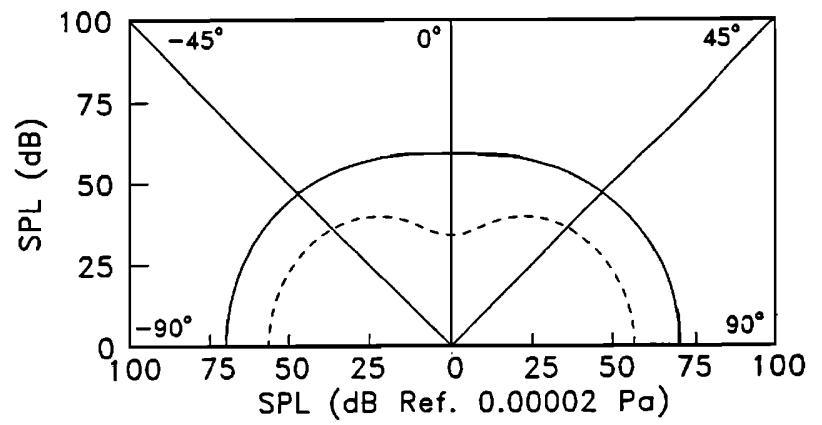

FIG. 6. Radiation directivity on the $x-z$ plane at $399 \mathrm{~Hz}$ : uncontrolled (----) and controlled $(\longrightarrow)$. 


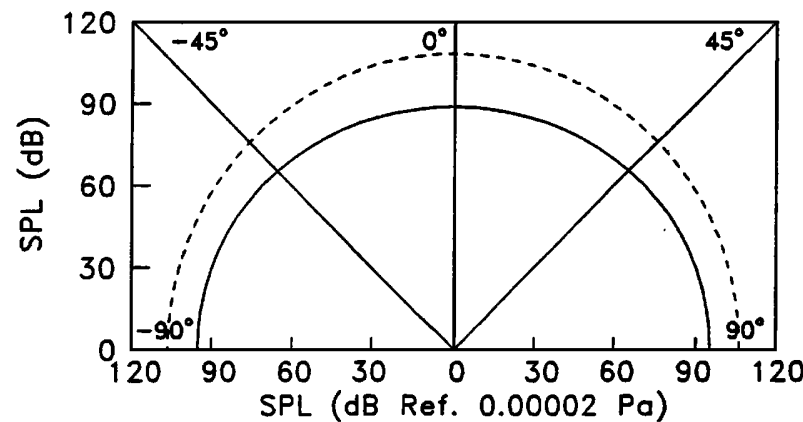

FIG. 7. Total radiation directivity on the $x-z$ and planes: uncontrolled (-.--) and controlled (-).

that yield the optimum modal parameter is not a key aspect of formulation presented here. However, for the sake of completeness and as well as clarity in the presentation, the design of the strain-induced actuator and sensor is presented in the Appendix.

\section{CONCLUSIONS}

A formulation has been presented for the design of adaptive structures for ASAC applications. The methodology makes use of the fundamental concept that sound radiation can be effectively reduced by changing the overall radiation efficiency of the structure. The formulation also takes advantage of the fact that the feedforward-control system changes the dynamic properties of the structure. The understanding and merging of these two phenomena lead to an efficient method for the design of actuators and sensors. The method is based on the premise that the optimum actuator and sensor will change the eigenstructure of the system such that the controlled response consists of a modal series of weak radiators. The control configuration is first defined in the modal domain by computing the optimum control and error modal parameters. Using this information, the physical actuator and sensor can be then constructed. This particular separation between the modal and physical domain offers the advantage that different transducers and configurations can be investigated with minimum computational effort. The design approach was illustrated for controlling the odd-odd modes on a simply supported plate problem driven by a point force. A SISO controller was designed and the results show excellent reduction over the whole spectrum.

\section{ACKNOWLEDGMENT}

The authors gratefully acknowledge the support of this work by the Office of Naval Research Grant No. ONRN00014-92-J-1170.

\section{APPENDIX: STRAIN-INDUCED ACTUATOR AND SENSOR DESIGN}

For the sake of completeness, in this appendix a procedure to design an structural actuator and sensor that yield the desired modal parameters is briefly presented. The actuator and error sensor are implemented with multiple piezoelectric PZT patches and a single PVDF film, respectively. Mechanical models that relate the transducer parameters, i.e., size, (a)

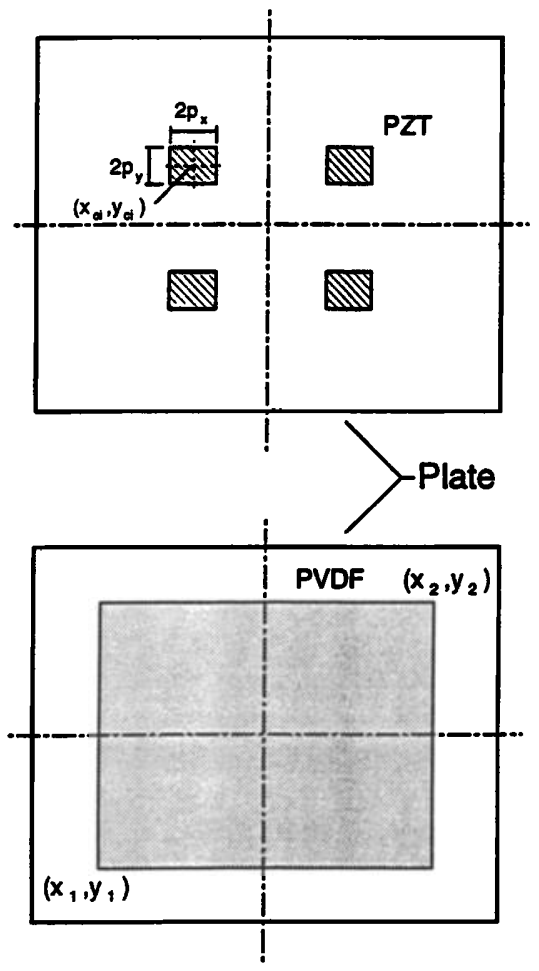

FIG. A1. Optimum (a) PZT control actuator and (b) PVDF film error sensor.

location, etc., to the modal control and error components are required. Assuming the control input consists of $M$ fixed-size PZT patches driven by the same control signal either in- or out-of-phase. Then, the $n$th modal control force can be shown to be given by ${ }^{26}$

$$
\begin{aligned}
u_{n}= & \sum_{i=1}^{M} P_{i} \frac{\gamma_{x}^{2}+\gamma_{y}^{2}}{\gamma_{x} \gamma_{y}}\left\{\cos \left[\gamma_{x}\left(x_{c i}+p_{x}\right)\right]-\cos \left[\gamma_{x}\left(x_{c i}-p_{x}\right)\right]\right\} \\
& \times\left\{\cos \left[\gamma_{y}\left(y_{c i}+p_{y}\right)\right]-\cos \left[\gamma_{y}\left(y_{c i}-p_{y}\right)\right]\right\},
\end{aligned}
$$

where $P_{i}= \pm 1$ indicates the relative phase between the PZT patches, $\left(x_{c}, y_{c i}\right)$ are the unknown center coordinates, and $2 p_{x}=0.1 L_{x}$ and $2 p_{y}=0.1 L_{y}$ are the fixed dimensions of the patch. The design variables are the relative phase and coordinates of the patches and are obtained by minimizing the cost function

$$
F\left(P_{i}, x_{c i}, y_{c i}\right)=\sum_{n=1}^{N}\left|\left(u_{n}\right)_{\mathrm{opt}}-u_{n}\right|^{2},
$$

where the optimum modal control forces $\left(u_{n}\right)_{\mathrm{opt}}$ are the values in Table II for the odd-odd modes and zero for all the others. The optimization process in Eq. (30) is continuous in the variables $\left(x_{c i}, y_{c i}\right)$ and discrete in the phase indicator $P_{i}$ and number of patches $M$. The solution of this optimization, carried out using IMSL routine DUNLSF, is straightforward and leads to the control configuration shown in Fig. A1(a). The control input for the SISO controller consists of four patches driven in phase by the control signal $U(\omega)$.

The error sensor was implemented with a single PVDF film patch. If needed multiple patches, whose electric output 
are summed to obtain the error signal, can be considered. The $n$th error modal component can be shown to be given as $^{12}$

$$
\begin{aligned}
\xi_{n}= & \left(e_{31} \frac{\gamma_{x}}{\gamma_{y}}+e_{32} \frac{\gamma_{y}}{\gamma_{x}}\right)\left[\cos \left(\gamma_{x} x_{1}\right)-\cos \left(\gamma_{x} x_{2}\right)\right] \\
& \times\left[\cos \left(\gamma_{y} y_{1}\right)-\cos \left(\gamma_{y} y_{2}\right)\right],
\end{aligned}
$$

where $\left(x_{1}, y_{1}\right)$ and $\left(x_{2}, y_{2}\right)$ are the lower left and upper right corner unknown coordinates, and $e_{31}$ and $e_{32}$ are charge per unit area in the $x$ and $y$ directions, respectively. The coordinates that determine the size and location of the PVDF film are obtained by minimizing

$$
F\left(x_{1}, y_{1}, x_{2}, y_{2}\right)=\sum_{n=1}^{N}\left|\left(\xi_{n}\right)_{\mathrm{opt}}-\xi_{n}\right|^{2},
$$

where the optimal modal error components $\left(\xi_{n}\right)_{\mathrm{opt}}$ are again obtained from Table II for the odd-odd modes and zero for all the other modes. The PVDF film error sensor is shown in Fig. A1(b).

${ }^{1}$ C. R. Fuller, "Apparatus and methods for global noise reduction," U.S. Patent No. 4,715,599 (1987).

${ }^{2}$ W. T. Baumann, W. R. Saunders, and H. H. Robertshaw, "Active suppression of acoustic radiation from impulsively excited structures,” J. Acoust. Soc. Am. 90, 3202-3208 (1991).

${ }^{3}$ L. Meirovitch and S. Thangjitham, "Active control of sound pressure," J. Vib. Acoust. 112, 237-244 (1990).

${ }^{4}$ C. R. Fuller, "Active control of sound transmission/radiation from elastic plates by vibrating inputs-I analysis," J. Sound Vib. 136, 1-15 (1990).

${ }^{5}$ V. L. Metcalf, C. R. Fuller, R. J. Silcox, and D. E. Brown, “Active control of sound transmission/radiation from elastic plates by vibration Inputs-II experiments," J. Sound Vib. 153, 387-402 (1992).

${ }^{6}$ J. P. Smith, C. R. Fuller, and R. A. Burdisso, "Control of broadband radiated sound with adaptive structures," in Proceedings of the SPIE 1993 North America Conference on Smart Structures and Materials, 31 Jan.-4 Feb. 1993, Albuquerque, NM (SPIE, Bellingham, 1993), pp. 587-597.

${ }^{7}$ B. T. Wang, R. A. Burdisso, and C. R. Fuller, "Optimal placement of piezoelectric actuators for active control of sound radiation from elastic plates," Proc. Noise-Con. 91, 267-275 (1991).

${ }^{8}$ C. R. Fuller, C. A. Rogers, and H. H. Robertshaw, "Active structural acoustic control with smart structures," Proceedings of the SPIE, Vol. 1170: Fiber Optic Smart Structures and Skins, Boston, MA, 338-358 (1989).

${ }^{9}$ C. R. Fuller, C. H. Hansen, and S. D. Snyder, "Experiments on active control of sound radiation using a piezoceramic actuator," J. Sound Vib. 150, 179-190 (1991).

${ }^{10} \mathrm{R}$. L. Clark and C. R. Fuller, "Experiments on active control of structurally radiated sound using multiple piezoceramic actuators," J. Acoust. Soc. Am. 91, 3313-3320 (1992).

${ }^{11}$ W. R. Saunders, H. H. Robertshaw, and C. A. Rogers, "Structural acoustic control for a shape memory alloy composite beam," AIAA paper 90-1090 CP (1990).

${ }^{12}$ R. L. Clark and C. R. Fuller, "Modal sensing of efficient acoustic radiators with polyvinylidene fluoride distributed sensors in active structural acoustic control approaches," J. Acoust. Soc. Am. 91, 3321-3329 (1992).

${ }^{13}$ R. L. Clark, C. R. Fuller, and R. A. Burdisso, "Design approaches for shaping polyvinylidene fluoride sensors in active structural acoustic control," in Proceedings of the Conference on Recent Advances in Adaptive and Sensory Materials and Their Applications, Blacksburg, VA (Technonic, Lancaster, PA, 1992), pp. 702-726.

${ }^{14}$ R. L. Clark, C. R. Fuller, B. R. Fogg, W. W. Miller, W. V. Vengsarkar, and R. O. Claus, "Structural acoustic control using fiber sensors and piezoelectric actuators," in Proceedings of the International Symposium and Exhibition on Active Materials and Adaptive Structures, Alexandria, VA (IOP, Philadelphia, 1991), pp. 547-552.

${ }^{15}$ R. L. Clark and C. R. Fuller, "Optimal placement of piezoelectric actuators and polyvinylidene fluoride (PVDF) error sensors in active structural acoustic control approaches," J. Acoust. Soc. Am. 92, 1521-1533 (1992).

${ }^{16}$ R. A. Burdisso and C. R. Fuller, "Theory of feedforward controlled system eigenproperties," J. Sound Vib. 153, 437-452 (1992).

${ }^{17}$ R. A. Burdisso and C. R. Fuller, "Feedforward controller design by eigenvalue assignment," AIAA J. Guidance Control 17(3), 466-472 (1994).

${ }^{18}$ R. A. Burdisso and C. R. Fuller, "Dynamic behavior of structural-acoustic systems in feedforward control of sound radiation," J. Acoust. Soc. Am. 92, 277-286 (1992).

${ }^{19} \mathrm{~J}$. T. Post, "Active control of the forced and transient response of a finite beam," Master's thesis, The George Washington University (October 1989).

${ }^{20}$ F. Fahy, Sound and Structural Vibration (Academic, Orlando, FL, 1985).

${ }^{21}$ SYSNOISE: Numerical analysis of acoustical and elasto acoustical problems (Dynamic Engineering, St. Louis, MO, 1992).

${ }^{22}$ C. E. Wallace, "Radiation resistance of a rectangular panel," J. Acoust. Soc. Am. 51, 946-952 (1972).

${ }^{23}$ D. C. Smith and R. J. Bernhard, "Computing acoustic design sensitivity information using boundary element methods," in Proceedings of The Eleventh International Conference on Boundary Element Methods in Engineering, Cambridge, MA, Vol. 2 (Springer-Verlag, New York, 1989), pp. 369-383.

${ }^{24}$ K. A. Cunefare and G. H. Koopmann, "Acoustic design sensitivity for structural radiators," J. Vib. Acoust. 114, 178-186 (1992).

${ }^{25} \mathrm{~K}$. A. Cunefare, "The minimum multimodal radiation efficiency of baffled finite beams," J. Acoust. Soc. Am. 90, 2521-2529 (1991).

${ }^{26}$ E. K. Dimitriadis, C. R. Fuller, and C. R. Rogers, "Piezoelectric actuators for distributed vibration excitation of thin plates," J. Vib. Acoust. 113, 100-107 (1989). 